\title{
Disclosure of diabetes status among Japanese police officers: a qualitative study
}

Shinomi Takahashi' ${ }^{1,2)}$, Yoshitaka Konda ${ }^{3)}$, S.Pilar Suguimoto ${ }^{1)}$, Patou Masika Musumari ${ }^{11}$, Teeranee Techasrivichien ${ }^{1)}$, Masako Ono-Kihara ${ }^{1)}$ and Masahiro Kihara ${ }^{1)}$

1) Graduate School of Medicine, Department of Global Health and Socio-epidemiology, School of Public Health Kyoto University, Japan

2) Inter-Graduate School Program for Sustainable Development and Survivable Societies, Kyoto University, Japan

Kyoto Police Hospital, Kyoto, Japan

\section{Background}

- Self-care of diabetes in the workplace poses a number of challenges; police work is an occupation with high risk of diabetes and where lifestyle changes may be difficult due to the nature of the job

- Social stigma is reported for diabetes type 1 and 2

- A previous study in Denmark reports that $23 \%$ of patients with type 2 diabetes mellitus (T2DM) had not disclosed their status to their employer

- Disclosure may be a key to appropriate management of T2DM and reduction of diabetes distress, but focus on this issue is limited

\section{Aim}

- To investigate the reasons for disclosure of diabetes status among different occupations, particularly among Japanese police officers

\section{Study setting and target population}

- Secondary hospital in Kyoto, Japan

- Study participants were male diagnosed with T2DM, aged 40-69, and current or retired from police workforce or other work activity

\section{Methods}

- Exploratory qualitative study using in-depth interviews guided by semi-structured questionnaires exploring knowledge of diabetes, work and work environment, and disclosure of diabetes, conducted from January to June 2017

- Purposive sampling during hospital appointment

- Interviews were audio-recorded, transcribed, manually coded, and analyzed through thematic analysis

- Ethical clearance from Kyoto University Committee for Research on Human Subjects and Kyoto Police Hospital ethical committee, no conflict of interest

- All participants provided written informed consent

\section{Results}

- This achieved a sample of 24 men having working history (7 police officers, 7 retired police officers and 10 other occupations)

- All participants, regardless of occupations, had disclosed their diabetes status to their manager out of necessity. However, some of the participants tend tc conceal their diabetes status from colleagues because they are concerned about the reaction of their colleagues and they experience no inconveniences.

- Regardless of occupation and reasons for disclosure, revealing their diabetes status in their workplace has had many positive effects. There were many common reasons for disclosure, however, the reasons among police officers were often explained based on the police culture and prior experiences as police officers.

- The participants reported having many negative beliefs related to T2DM before being diagnosed.

\section{Theme 1: Negative beliefs about diabetes}

- Diabetes is a disease that can only be avoided with a healthy lifestyle

- Diabetes is a lifelong disease and using insulin is like a death sentence

- Word for diabetes in Japanese “糖尿病(tou-nyou-byou)" evokes negative impression; “糖” means sugar, “尿” means urine, and “病” means disease.

\section{Theme 2: Uncertainty of diabetes after diagnosis}

- Doubtful about diagnosis even after diagnosis of T2DM

- For asymptomatic patients, having regular appointments, having blood tests and prescription are the most important factors to maintain awareness of T2DM

\section{Theme 3: Features of police officers}

\section{- Work is the priority until retirement}

- Cannot talk about non-work related topics in the workplace, including health

- Prefer high-calories food and alcohol

\section{Theme 4: Differentiation of decision to disclose in the workplace}

\section{Disclosure of diabetes status to manager}

- By necessity

"Of course I disclosed to my boss, because police is such a hierarchy society, we have to report our diseases." (42y, Police)

\section{Disclosure of diabetes status to colleagues \\ - By chance}

"Because the morning after night shifts, I need to take medication, so conceal of diabetes felt wrong, so I told at that time." (54y, Police)

\section{- Voluntary}

-Because of need better management of blood sugar level

-Because to promote well-being at the workplace using their personal experience "When I was a police instructor, I explained my disease because I didn't want police officers to get diabetes like me." (66y, Retired police)

\section{- Concealment is a burden}

"There is no advantage to hide diabetes, right? ... conceal of anything becomes a burden for us... so I disclose." (59y, Police)

\section{Concealment from colleagues}

\section{Diabetes is an unwanted topic}

"It was the mood..., we could say "I couldn't go drinking because of lack of money" but we couldn't say "I didn't go drinking because of diabetes!"," (66y, Retired police)

\section{No benefits in terms of workload}

"I don't talk about diabetes in the workplace, because..., if I disclose my diabetes status, nothing will change. It will not reduce my tasks." (42y, Police)

\section{To avoid making the company staff anxious}

"I don't talk about my diabetes in front of my staff, because leaders having such kind of disease will make staff concern...I'm OK whether I disclose my diabetes status, but my concern is how will employee take it." (49y, Non-police)
Participants characteristics

\begin{tabular}{|c|c|c|c|c|}
\hline & & $\begin{array}{c}\text { Police } \\
\text { officer } \\
(n=7)\end{array}$ & $\begin{array}{c}\text { Retired } \\
\text { police officer } \\
\quad(n=7)\end{array}$ & $\begin{array}{c}\text { Non-police } \\
(n=10)\end{array}$ \\
\hline \multirow[t]{3}{*}{ Age } & $40-49$ years old & 1 & - & 2 \\
\hline & 50-59 years old & 6 & - & 5 \\
\hline & $60-69$ years old & - & 7 & 3 \\
\hline \multirow{3}{*}{$\begin{array}{l}\text { Current } \\
\text { treatment }\end{array}$} & Diet only & 2 & 1 & 1 \\
\hline & Medication \& Diet & 5 & 5 & 7 \\
\hline & Medication \& Insulin & 0 & 1 & 2 \\
\hline \multicolumn{2}{|c|}{ Diabetes duration (years) } & $1-12$ & $1-25$ & $2-15$ \\
\hline \multicolumn{2}{|c|}{ Disclosure to manager } & 7 & 7 & 10 \\
\hline & to colleagues & 5 & 6 & 8 \\
\hline \multicolumn{2}{|c|}{ Conceal from manager } & 0 & 0 & 0 \\
\hline \multicolumn{2}{|c|}{ from colleagues } & 2 & 1 & 2 \\
\hline
\end{tabular}

\section{Theme 5: Effects of disclosing}

\section{- Encourage others to disclose their status}

when I said: I was hospitalized because of diabetes. My colleague replied: I was taking medicine for diabetes too." (56y, Police)

\section{- Peer support}

"After disclosing my diabetes status, when I ate a snack, colleagues of mine said something to take care. I opened up because it is better to manage my diabetes." (54y, Police)

"People around me don't force me to drink any more." (58y, Non-police)

\section{- Receiving information related to diabetes}

"Information about diabetes comes to me because of disclosure." (60y, Non-police)

\section{Conclusions}

- Disclosure of T2DM might have health promoting effects in the workplace including police workplace. However, considering that the reasons to disclose among police officers is influenced by characteristics of their job, intervention in this group should be developed respecting these characteristics.

- Reducing the negative beliefs about diabetes in society would be important to encourage disclosure of diabetes in the workplace, which in turn could facilitate its management. 